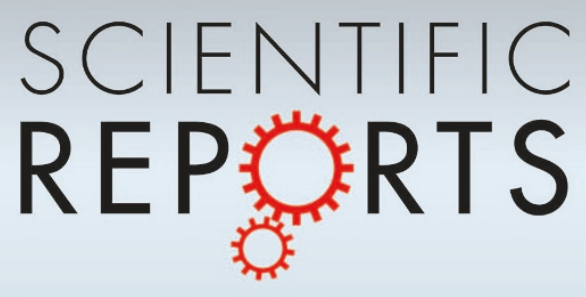

OPEN

SUBJECT AREAS:

BIOMEDICAL

ENGINEERING

CARBON NANOTUBES AND

FULLERENES

OPTICAL TECHNIQUES

APPLIED PHYSICS

Received

5 September 2012

Accepted

6 November 2012

Published

18 December 2012

Correspondence and requests for materials should be addressed to

L.J.G. (guo@umich.

edu)

* Current address: Harvard Medical School, Wellman

Center for

Photomedicine,

Massachusetts

General Hospital,

Boston, MA 02114

USA

\title{
Carbon-Nanotube Optoacoustic Lens for Focused Ultrasound Generation and High-Precision Targeted Therapy
}

\author{
Hyoung Won Baac ${ }^{*}$, Jong G. Ok ${ }^{2}$, Adam Maxwell ${ }^{3}$, Kyu-Tae Lee', Yu-Chih Chen ${ }^{1}$, A. John Hart², \\ Zhen $\mathrm{Xu}^{3}$, Euisik Yoon' \& L. Jay Guo ${ }^{1,2}$
}

'Department of Electrical Engineering and Computer Science, The University of Michigan, Ann Arbor, MI 48109 USA, ${ }^{2}$ Department of Mechanical Engineering, The University of Michigan, Ann Arbor, Ml 48109 USA, ${ }^{3}$ Department of Biomedical Engineering, The University of Michigan, Ann Arbor, MI 48109 USA.

We demonstrate a new optical approach to generate high-frequency $(>15 \mathrm{MHz})$ and high-amplitude focused ultrasound, which can be used for non-invasive ultrasound therapy. A nano-composite film of carbon nanotubes (CNTs) and elastomeric polymer is formed on concave lenses, and used as an efficient optoacoustic source due to the high optical absorption of the CNTs and rapid heat transfer to the polymer upon excitation by pulsed laser irradiation. The CNT-coated lenses can generate unprecedented optoacoustic pressures of $>50 \mathrm{MPa}$ in peak positive on a tight focal spot of $75 \mu \mathrm{m}$ in lateral and $400 \mu \mathrm{m}$ in axial widths. This pressure amplitude is remarkably high in this frequency regime, producing pronounced shock effects and non-thermal pulsed cavitation at the focal zone. We demonstrate that the optoacoustic lens can be used for micro-scale ultrasonic fragmentation of solid materials and a single-cell surgery in terms of removing the cells from substrates and neighboring cells.

(a) igh-amplitude focused ultrasound can provide localized perturbation in liquids and tissues by inducing shock, acoustic cavitation, and heat deposition within focal volumes ${ }^{1-4}$. Such mechanical and thermal disturbances have been widely used to deliver targeted impacts to cells and tissues for biomedical therapy: for example, trans-membrane drug delivery (e.g. trans-dermal and blood-brain barrier opening $)^{5-7}$, neural activity modulation in brain ${ }^{8,9}$, and thrombolysis ${ }^{10}$, often relying on acoustic cavitation or externally injected micro-bubbles. Remarkable progress have been made in clinical kidney-stone fragmentation ${ }^{11,12}$ as well as ablation-based tumor therapy under high-intensity focused ultrasound (HIFU) ${ }^{13-15}$. Moreover, cavitation-based ultrasound therapy such as histotripsy has shown some success as a new invasive mechanical ablation tool ${ }^{16}$.

Although the above beneficial effects have been confirmed over a broad range of biomedical applications, their focal dimensions are insufficiently large: typically $>2 \mathrm{~mm}$ in a lateral plane and often $>10 \mathrm{~mm}$ in an axial plane. This is because the focused ultrasound has been generated using low-frequency piezoelectric transducers (a few $\mathrm{MHz})^{14}$. Moreover, the low-frequency pressure waves necessitate large lens sizes on the order of several centimeters which are not proper for intra-operative applications. High-frequency ultrasound (tens of $\mathrm{MHz}$ ) would provide obvious advantages on spatial and temporal confinement, opening numerous opportunities for highaccuracy cell therapy as well as ablation-treatment over single tissue layers and micro-vasculatures. It should be also noted that tumors are very often grown adjacent to a vital blood vessel that should be kept intact, and can be hardly addressed by the bulky focal spots in a selective manner. Therefore, high-precision ablation is essential for use in surgery.

However, it is challenging to achieve therapeutic pressure amplitudes in the high-frequency regime $(>10 \mathrm{MHz})$ : for example, stronger tensile pressure $(P)$ is required at higher frequency $(f)$ to induce the acoustic cavitation (i.e. $P \propto f^{1 / 2}$ approximately $\left.{ }^{17}\right)$ which can create significant impacts upon adjacent media through liquid micro-jets and shock waves due to bubble collapse. Furthermore, such high pressure must be achieved at the focal spot in spite of severe acoustic attenuation for the high-frequency components $\left(2.2 \times 10^{-3} \mathrm{~dB} /\left(\mathrm{cm} \times \mathrm{MHz}^{2}\right)\right.$ in water). The acoustic cavitation can be facilitated by heat deposition through repetitive pulses, but thermal heating should be avoided in many cases of cell therapy applications (e.g. gene therapy) because cellular metabolism is easily transformed by a slight temperature change. A single pulsed cavitation without heat deposition would be useful in these applications. 
Alternatively, the high-frequency ultrasound has been generated using pulsed laser irradiation on light-absorbing materials which in turn causes thermo-elastic volume expansion. This method of optoacoustic generation could easily reach several tens of $\mathrm{MHz}$, and even $\mathrm{GHz}^{18-20}$ in the acoustic frequency: however, poor energy conversion efficiency has been a major drawback, resulting in weak pressure amplitudes. The advantage of high frequency is further compromised by the frequency-dependent attenuation over long-range propagation. Due to these limitations, optoacoustic pressure as a high-frequency source has not been shown to be feasible for deeptissue imaging as well as therapeutic purposes requiring high pressure amplitude of tens of $\mathrm{MPa}$.

We demonstrate laser-generated focused ultrasound (LGFU) as a new modality which can produce high-frequency $(>15 \mathrm{MHz})$ and unprecedented optoacoustic pressure of $>50 \mathrm{MPa}$ from a singleelement lens of only $6 \mathrm{~mm}$ in diameter. The focused ultrasound is generated by using a uniquely designed optoacoustic transmitter, made of carbon-nanotube (CNT)-polymer composites, which is formed on a concave surface that directly enables acoustic focusing. Such high-amplitude ultrasound, going into a therapeutic regime, is obtained due to an efficient energy conversion process by the CNTcomposites and a high focal gain in the optoacoustic lens platform. The acoustic performance of the LGFU is temporally and spatially characterized at the focal spot, which is as small as $75 \mu \mathrm{m}$ in lateral and $400 \mu \mathrm{m}$ in axial directions. Remarkably, it is shown that the LGFU produces powerful shock waves and single-pulsed cavitation, both of which can be used as strong sources of mechanical disruption. These enable micro-scale lithotripsy and high-precision targeted cell therapy. We demonstrate that the spatial dimension of the mechanical disruption can be controlled from $6 \sim 15 \mu \mathrm{m}$ up to $300 \sim 400 \mu \mathrm{m}$ within the focal zone.

\section{Results}

Nano-composite optoacoustic transmitters. The optoacoustic source was devised to have high optical absorption, efficient heat transduction, and high thermal expansion. For the high optical absorption, we used multi-walled CNTs which were grown on fused silica substrates by chemical vapor deposition (CVD). The CNT length and areal density were controlled to have an optical extinction of $60 \sim 70 \%$ on the substrate. Then, we further increased the optical extinction up to $>85 \%$ by depositing a gold ( $\mathrm{Au}$ ) layer of $20 \mathrm{~nm}$ onto the CNT films. Finally, an elastomeric polymer, polydimethylsiloxane (PDMS), was spin-coated onto the Au/CNT layer. Fig. 1(a) and 1(b) show cross-sectional views of the Au-coated CNTPDMS composite layer fabricated on the concave lens.

The nano-scale dimensions and thermal properties of the CNTs were essential to realize the efficient optoacoustic transmitter. Rapid heat diffusion to a surrounding medium is one of the key characteristics of the nano-particles. For a given heat diffusion time determined by the characteristic dimension of the nano-structure, we can estimate a fraction of the thermal energy $\eta$ within the absorbers after the laser ${ }^{21}$,

$$
\eta=\frac{\tau_{H D}}{\tau_{L}} \times\left[1-\exp \left(-\frac{\tau_{L}}{\tau_{H D}}\right)\right]
$$

where $\tau_{H D}$ and $\tau_{L}$ are the heat diffusion time and the laser pulse duration (Fig. 1(c)). Considering the CNT as a cylinder with diameter $d$, the diffusion time is obtained as $\tau_{H D}=d^{2} / 16 \chi$ where $\chi$ is the thermal diffusivity of the surrounding medium. This results in $\tau_{H D}<$ $0.4 \mathrm{~ns}$ for the Au-coated CNT strand ( $\sim 25 \mathrm{~nm}$ in diameter) surrounded by the PDMS $\left(\chi=1.06 \times 10^{-7} \mathrm{~m}^{2} / \mathrm{s}\right)$. This is much faster than the temporal width of the laser pulse (6 ns), suggesting that the negligible energy remains within the $\mathrm{CNT}(\eta=0.06)$ after the optical pulse excitation. This means that, as soon as the CNTs are heated by the light absorption, they give out most of the thermal energy to the surrounding elastomeric polymer which can cause instantaneous thermal expansion with high amplitudes. The PDMS has a high thermal coefficient of volume expansion, $0.92 \times 10^{-3} \mathrm{~K}^{-1}$, which is 4.5 fold higher than water $\left(0.21 \times 10^{-3} \mathrm{~K}^{-1}\right)$, and $>20$ fold higher than typical metals (e.g. gold $\left.\sim 0.04 \times 10^{-3} \mathrm{~K}^{-1}\right)$. Our approach to simultaneously achieve rapid and large volume expansion using the CNT nano-composite is clear distinction from the use of micro-scale optical absorbers commonly used as optoacoustic imaging contrast agents. Comparatively, for a micro-scale cylinder of $1 \mu \mathrm{m}$ in diameter, most of the generated heat is confined $(\eta=0.99)$ after the same pulse duration of $6 \mathrm{~ns}$. Therefore, the volume deformation is dominated by the optical absorbers themselves.

Optoacoustic lenses with high focal gains. For acoustic focusing, the CNT-PDMS composite transmitters were produced on a concave surface. We used fused silica optical lenses as the concave substrate. Then, we took the unique advantage of the CNTs which can be directly grown on arbitrary shaped surfaces ${ }^{22}$. Because the growth of CNT films is conformal to the surface, we could use spherical lenses with deep curvatures (i.e. low $f$-number) to allow high focal gains.

We used two lenses for experimental demonstration. The first lens has 5.5- $\mathrm{mm}$ radius of curvature and 6- $\mathrm{mm}$ diameter (named as type I), and the second has $11.46-\mathrm{mm}$ curvature and $12-\mathrm{mm}$ diameter (type II). The focal gain $G$ of spherical lens can be represented as a ratio of the pressure at the focus to the pressure on the spherical surface where the source layer is located ${ }^{23}$ :

$$
G=\frac{2 \pi f}{c_{0}} r\left(1-\sqrt{1-\frac{1}{4 f_{N}^{2}}}\right)
$$

Here, $f, c_{o}, r$, and $f_{N}$ are the acoustic frequency, the ambient sound speed, the radius of curvature, and the $f$-number which is defined as the ratio of the radius of curvature to the lens diameter. As both lenses have low $f$-numbers, 0.92 (type I) and 0.96 (type II), their focal gains are significantly greater than the typical HIFU transducers with $f_{N}>2$. According to the equation (2), the gain $G$ at $f_{N}=0.92$ can be $5-11$ fold higher than those at $f_{N}=2-3$. To account for the acoustic attenuation in water, we can obtain effective focal gains $G_{\text {eff }}$ by multiplying $G$ with a frequency-dependent attenuation coefficient which is $2.2 \times 10^{-3} \mathrm{~dB} /\left(\mathrm{cm} \times \mathrm{MHz}^{2}\right)$. Therefore, for the two chosen lenses, we estimate $G_{\text {eff }}$ (type I) $\approx 54$ and $G_{\text {eff }}$ (type II) $\approx 100$ at $15-\mathrm{MHz}$ frequency.

Characterization of LGFU. Using the type I optoacoustic lens, we observed strong shock waves at the lens focus, as measured using a single-mode fiber-optic hydrophone (Fig. 1(c)). Experimental waveforms of the LGFU are shown in Fig. 2(a). In principle, optoacoustic pressure waveforms should be close to the time-derivative of the original laser pulse (i.e. Gaussian) due to linear wave propagation in a far-field regime ${ }^{24}$. However, the measured waveform was highly asymmetric near the focal point (we assume radius-of-curvature of lens $\approx$ focal length, i.e. $z_{f}=5.5 \mathrm{~mm}$ ). The asymmetric distortion is caused by nonlinear propagation of the finite-amplitude pulse which leads to the development of pronounced shock front in the positive phase and longer trailing in the negative phase. This is similar to that observed in typical shockwave lithotripsy. We confirmed that the distortion only develops within the focal zone as a symmetric waveform is clearly observed in the pre-focal zone at $z=z_{f}-0.3=$ $5.2 \mathrm{~mm}$. The peak positive pressure of the focal waveform of Fig. 2(a) corresponds to $\sim 22 \mathrm{MPa}$ and the negative is $\sim 10 \mathrm{MPa}$, both of which were determined after excluding the bandwidth effect of the fiber (the detail of hydrophone sensitivity is described in the experimental section). These were obtained for the laser energy of $\sim 12 \mathrm{~mJ} /$ pulse on the lens surface (laser fluence $=42.4 \mathrm{~mJ} / \mathrm{cm}^{2}$ / pulse). Note that the maximum-available laser energy, which does not cause transmitter damage, is 7 -fold higher. For the focal waveform shown in Fig. 2(a), we estimated the optoacoustic conversion efficiency ${ }^{25}$ that is expressed as 


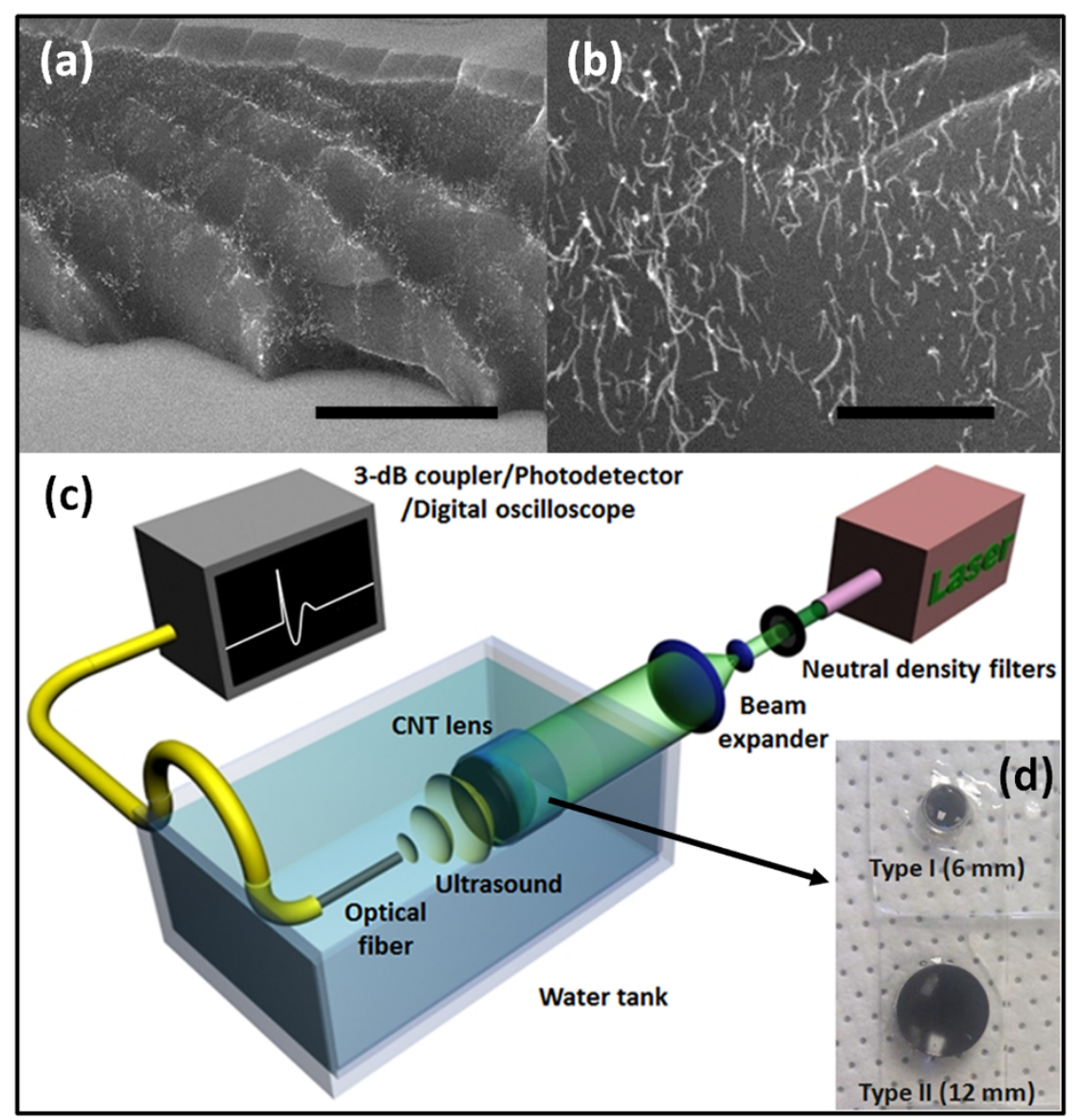

Figure 1 Optoacoustic lenses and measurement setup. The cross-sectional views of the gold-coated CNT-PDMS composite layer are shown in (a) (scale bar $=10 \mu \mathrm{m})$ and $(\mathrm{b})($ scale bar $=1 \mu \mathrm{m})$, taken by the scanning electron microscopy. The type II lens shown in (d) was used for the SEM characterization of (a) and (b). The layer thickness is $\sim 16 \mu \mathrm{m}$. The PDMS is completely infiltrated among the CNT network as shown in (b); (c) An experimental setup for the LGFU characterization. The 6-ns pulsed laser beam is expanded $(\times 5)$ and then irradiated onto the transparent side of the CNT lens $($ detailed explanation in the method section). The LGFU was optically detected by scanning the single-mode fiber-optic hydrophone. The optical output was 3 -dB coupled and transmitted to the photodetector with an electronic bandwidth of $75 \mathrm{MHz}$; (d) Two CNT lenses used in this work. The CNTs were grown on the concave side of the plano-concave fused silica lenses.

$$
K=\frac{\left|\frac{1}{T} \int_{T} P(t) d t\right|}{\frac{1}{T} \int_{T} I(t) d t}
$$

where $P(t), I(t)$, and $T$ are the pressure, the optical intensity, and the temporal period $(=50 \mathrm{~ms})$. For the time-averaged optical intensity of $0.85 \mathrm{~W} / \mathrm{cm}^{2}$, we obtain $K=1.4 \times 10^{-3} \mathrm{~Pa} /\left(\mathrm{W} / \mathrm{m}^{2}\right)$. This efficiency is two orders of magnitude higher than those of Cr films $\left(\sim 10^{-5}\right)$.

Next, we investigated the pressure amplitudes by increasing the excitation laser energy. We measured the focal waveforms from the type I lens, and then determined the peak positive and peak negative pressure. As shown in Fig. 2(b), the positive peak values were saturated to $\sim 57 \mathrm{MPa}$ over the high laser energy level. The saturation is attributed to the measurement reaching the bandwidth limit of the hydrophone. As a result, the highest frequency components of the shock wave cannot be accurately detected. For the negative amplitudes, the peak values could not be accurately determined at the high laser energy level. This is due to involvement of acoustic cavitation on the fiber surface which distorts the negative waveforms. In Fig. 2(b), the measurable negative peak values reach $\sim 13.3 \mathrm{MPa}$ at the laser energy of $14 \mathrm{~mJ} /$ pulse which is defined as the threshold laser energy $E_{t h}$ to induce the cavitation. However, by extrapolation, we estimate that $>25 \mathrm{MPa}$ can be reached in the negative phase.

Fig. 2(c) shows the corresponding frequency spectra of the LGFU. These experimental spectra include frequency bandwidth effects of the detector. Due to the finite diameter of the optical fiber $(125 \mu \mathrm{m})$, its sensitivity has a primary peak around $12 \mathrm{MHz}$ and higher-order peaks at 36 and $60 \mathrm{MHz}^{26}$. These are confirmed for the frequency spectrum of the symmetric waveform at the pre-focal zone ( $z=$ $5.2 \mathrm{~mm})$. In contrast, the spectrum at the focal zone $(z=5.5 \mathrm{~mm})$ shows significant enhancement over the high-frequency amplitudes $(>15 \mathrm{MHz}$ ), which manifests in the time domain as the distorted waveform with steep shock front. This also moved the experimental center frequency $f_{C}$ to $\sim 15 \mathrm{MHz}$. Due to the strong nonlinear distortion, the higher-order spectral peaks were also observed around $2 f_{C}, 3 f_{C}$, and $4 f_{C}$.

The high-frequency characteristics of the optoacoustic focusing were further manifested spatially as a tightly focused spot. In Fig. 2(d) and 2(e), we show the focal profiles of the type I lens within the lateral plane and along the axial direction, respectively. We achieved tight focal widths of $75 \mu \mathrm{m}$ in the lateral and $400 \mu \mathrm{m}$ in the axial directions which were determined by $6-\mathrm{dB}$ positive amplitudes. For the type II lens with two-fold longer focal length but the similar $f$-number, the lateral and axial widths broadened slightly to $100 \mu \mathrm{m}$ and $650 \mu \mathrm{m}$ because of acoustic attenuation of the high-frequency components over the long propagation distance.

LGFU-induced acoustic cavitation. In the experiment performed for Fig. 2(b), the measurable maximum negative pressure was 


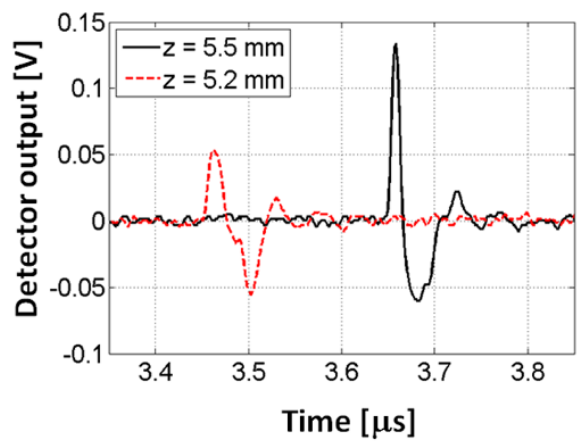

(a)

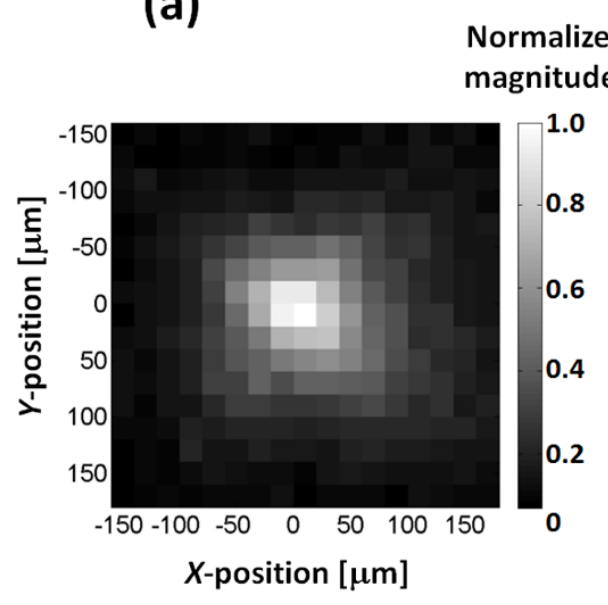

(d)

Normalized .

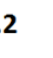

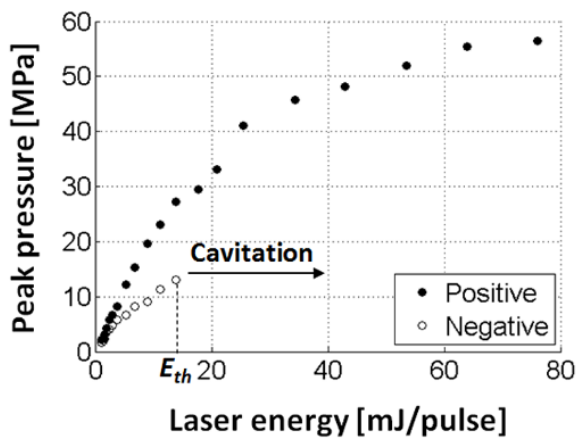

(b)

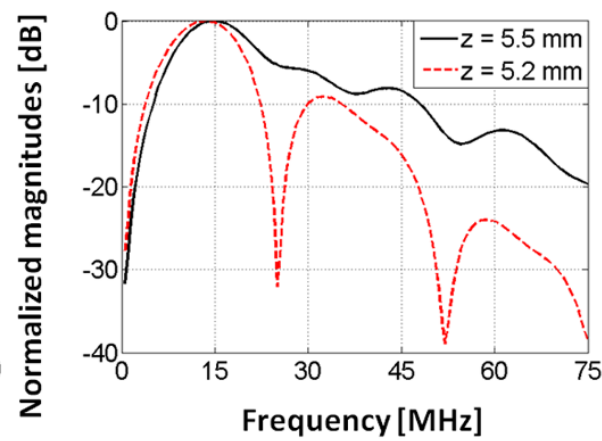

(c) 


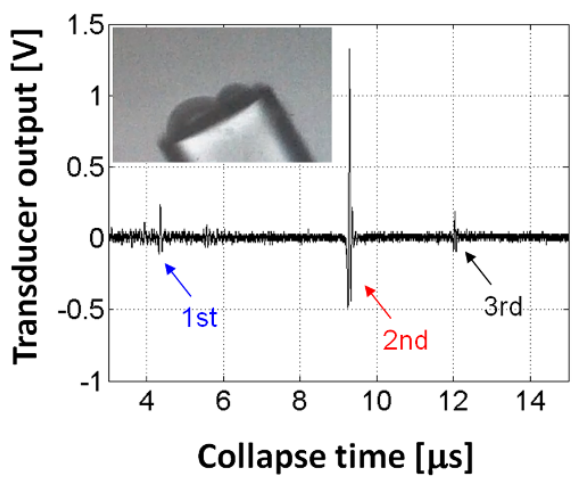

(a)

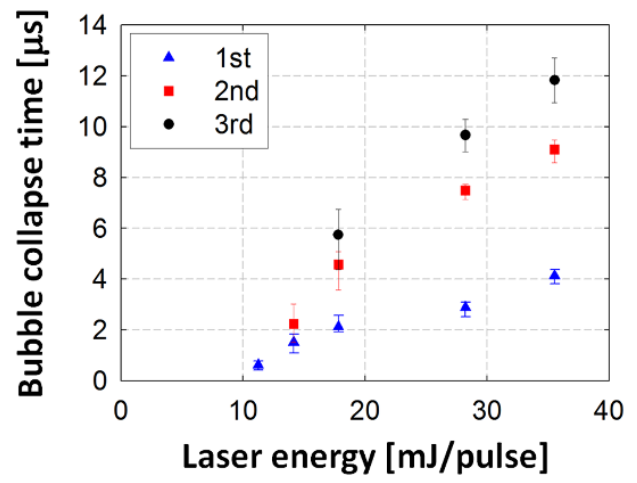

(b)

Figure 3 Measurement of the collapse time of cavitation bubbles generated by a single LGFU pulse. (a) Individual collapse events are detected in the time-domain. Each arrow (1st, 2nd, and 3rd) indicates the pressure signal emitted from each collapse. The inset shows the cavitation bubbles formed on the fiber surface (fiber diameter $=125 \mu \mathrm{m}$ ). Note that the image was separately taken by the high-speed camera (not exactly the same moment with the signal trace here). (b) The bubble collapse times are plotted as a function of the laser energy. No cavitation signal was observed for lower than $10 \mathrm{~mJ} / \mathrm{pulse}$ which is the cavitation threshold for the type II lens.

created by delivering $>1000$ pulses (or $>50 \mathrm{sec}$ ). Under this saturated exposure condition, the destroyed spot was $300 \sim 400 \mu \mathrm{m}$ in size. For comparison, we also produced line patterns by short exposure to the LGFU. Here, we translated the stone laterally at $\sim 0.4 \mathrm{~mm} / \mathrm{sec}$ while fixing the ultrasonic focal spot. This gave $<30$ pulses delivered at any position (or $1.5 \mathrm{sec}$ dwell time) along the lines of the stone surface. The destroyed line width was $\sim 150 \mu \mathrm{m}$. This dimension is an order of magnitude smaller than typically achieved by low-frequency transducers.

We note that the width of the damage zone can be controlled by changing the laser energy and thereby manipulating the high-pressure area at the focal spot. The damage zone is determined by where the pressure amplitude is higher than a specific threshold level, i.e. depending on the hardness and acoustic impedance of the solid. In this experiment, the widths of the single indentation and the lines were larger than the FWHM of the type II lens $(100 \mu \mathrm{m})$. This is because the focal pressure was sufficiently high, so that even the surrounding area (wider than the FWHM spot) was subject to pressure exceeding the threshold for the ultrasonic damage. By reducing the LGFU amplitude, we also confirmed that the disrupted dimension can be much smaller than the FWHM. As shown in Fig. 4(b), we could produce a micro-hole on the PMMA film. Here, we used the polymer film coated on the glass substrate for microscopic visualization. The micro-hole was produced by a single LGFU pulse as a micro-scale polymer piece was torn off from the substrate by the highly focused ultrasound. A typical dimension of the micro-hole was $6 \sim 15 \mu \mathrm{m}$ for laser energy of $10 \sim 15 \mathrm{~mJ} /$ pulse. This is defined as the minimum size of a feature that can be machined using the current LGFU system.

Next, we investigated cavitational contribution in the fragmentation process by using a high-speed recording system on an inverted microscope (supplementary video clip). Fig. 4(c) shows the focal spot image including a cloud of micro-bubbles formed on the polymer film. The LGFU amplitude was $\sim 40 \mathrm{MPa}$ in the peak positive and higher than the cavitation threshold in the negative (laser energy: $20 \sim 25 \mathrm{~mJ} /$ pulse). As the LGFU-treated spot is scanned from the bottom to the top direction in Fig. 4(c), it leaves many bright dots due to the torn-off polymer micro-pieces. Fig. 4(d) is taken in the same spot but $\sim 1.5$ second after the image of Fig. 4(c). The prolonged exposure produced more micro-cracks than in Fig. 4(c). Because the damaged regions including such micro-cracks facilitate the cavitation process (indicated by the black arrows), the fragmentation was expedited by the collapse of the collateral micro-bubbles in contact with the polymer.
Targeted cell removal in high precision. The high-precision micromachining capability of LGFU was further exploited in a demonstration of single-cell surgery by removing individual cells from substrates and from neighboring cells (supplementary video clip). Fig. 5(a) shows human ovarian cancer cells (2 days after inoculation) before the ultrasound exposure. The cells were cultured on a PMMA-copolymer film that was used as an adhesion layer on the glass substrate. Fig. 5(b) shows the result of LGFU exposure for the laser energy $E \approx 1.2 E_{t h}(12 \sim 13 \mathrm{~mJ} / \mathrm{pulse})$. The

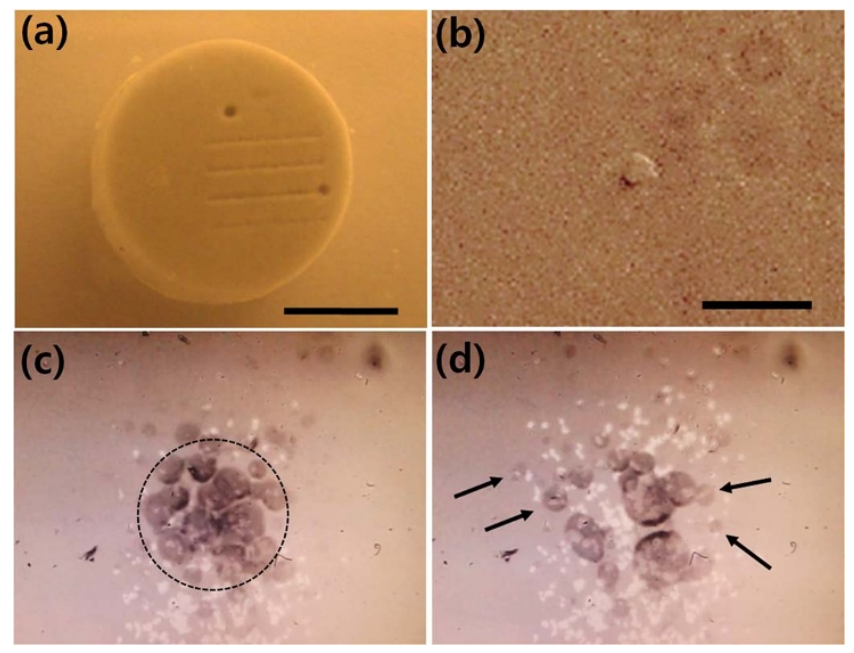

Figure $4 \mid$ Micro-scale fragmentation of the solid materials by the LGFU. (a) The model kidney stone ( scale bar $=4 \mathrm{~mm}$ ) was treated by the LGFU. $>1000$ pulses were delivered on the single spot on the top $(300 \sim 400 \mu \mathrm{m}$ in diameter), and $<30$ pulses to each position of the line patterns ( $\sim 150 \mu \mathrm{m}$ in width); (b) A single micro-hole on the polymer film (dented at the center) was produced by a single LGFU pulse (scale bar $=20 \mu \mathrm{m})$. A polymer micro-piece was torn off from the substrate; (c) and (d) Highspeed microscopic images of fragmentation process on the polymer-coated glass substrate. The transient bubbles were visualized by the high-speed camera. The focal spot of the LGFU is marked by the dotted circle in (c) (125 $\mu \mathrm{m}$ in diameter). The LGFU spot in (c) moves from the bottom to the top direction, leaving many bright dots which correspond to the polymerremoved regions. The same position on the polymer film is shown in (c) and (d) in the identical scale, but (d) is taken after the continued LGFU exposure of $\sim 1.5$ second. The black arrows in (d) indicate the preferential bubble formation along the micro-cracks. 

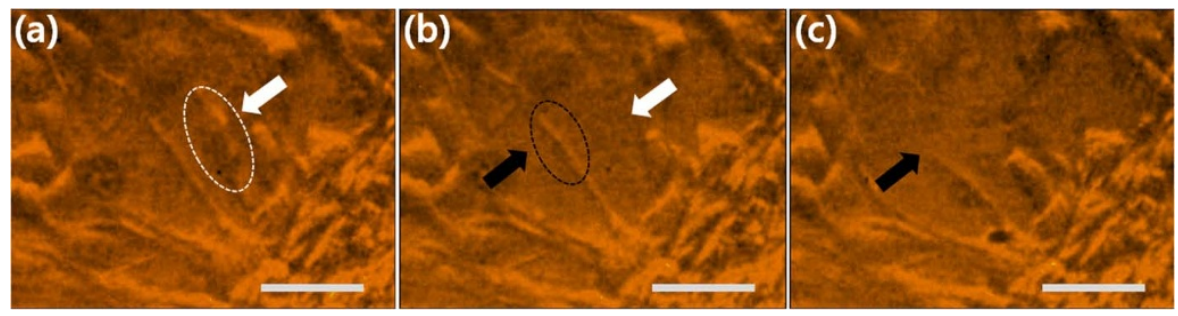

Figure $5 \mid$ Targeted cell removal by the LGFU (scale bar $=\mathbf{2 0} \boldsymbol{\mu m}$ ). The images were captured from a video clip (supplementary): (a) Cultured ovarian cancer cells (SKOV3) before ultrasound exposure. The white arrow indicates the single cell to be detached by the LGFU; (b) After the LGFU exposure, the single cell was selectively removed (indicated by the white arrow). As a next target, the cell-cell junction is indicated by the black arrow; (c) As the LGFU spot was moved to the black-dotted region, the cellular interconnection was severed.

LGFU could selectively remove the single cell within the white dotted region. Continuously, the LGFU spot was slightly moved to the adjacent region (black dotted) where the cell-cell junction was formed beforehand. As shown in Fig. 5(c), the single cellular junction was ruptured exactly as intended using the LGFU. Several to tens of LGFU pulses were used to detach the cells, depending on the individual cell shape on the substrate and the formation of cellular network with the surrounding cells. Here, we were able to locally control damage to the cells with $<25 \mu \mathrm{m}$ which is smaller than the FWHM of the focal spot. However, under the higher pressure regime with laser irradiation of $>40 \mathrm{~mJ} /$ pulse, a cluster of cells $(>100 \mu \mathrm{m}$ in diameter) could be removed.

\section{Discussion}

We demonstrated that the focused nano-composite optoacoustic transmitters that can generate sufficient pressure amplitudes to induce shock waves and cavitation at tight focal spots. However, the experimental values reported here are certainly not the ultimate limits of the LGFU approach as these limits depend on lens designs, pulsed lasers for optical excitation, and nano-composite properties. One of the key advantages in the LGFU is the compact dimension of the transmitters. As we have achieved the pressure amplitudes of $>50 \mathrm{MPa}$ from the lens with 6-mm diameter, we expect that a few tens of $\mathrm{MPa}$ is still available from smaller lenses $(<3 \mathrm{~mm})$ which accommodate intra-vascular and intra-operative applications. The output pressure can be further enhanced by improving the composite properties and using lenses with lower $f$-numbers. This offers potential for use of LGFU transmitters for non-contact mechanical surgery in endoscopic platforms.

As ultrasonic transmitters, nano-scale optical absorbers including $\mathrm{CNTs}^{20}$, planar gold nano-structures ${ }^{19,29}$, and synthesized gold nanocomposites $^{30}$ have been introduced due to their efficient energy conversion under pulsed laser irradiation and along with their excellent high-frequency performances. Especially, CNT-PDMS composite films have exhibited several-fold higher optoacoustic efficiency than those of typical gold-nanostructures ${ }^{20}$ over a broad range of frequency up to $120 \mathrm{MHz}$. Moreover, as the current LGFU requires relatively high laser energy on the order of tens of $\mathrm{mJ} /$ pulse, a thermal damage threshold of the source material is an important factor which determines the maximum-available laser energy. We experimentally confirmed that the CNT-PDMS composite film has a damage threshold of $280-300 \mathrm{~mJ} / \mathrm{cm}^{2}$ for a 6-ns laser pulse. This is $7-8$ fold higher than those of PDMS-coated gold-nanostructures and thin metal films measured in the same configuration.

The LGFU performance, in terms of pressure amplitude, intensity, frequency spectrum, and focal spot sizes, can be controlled externally by the excitation lasers. Here, we used 6-ns in the laser pulse width, $20 \mathrm{~Hz}$ in the repetition rate, and tens of $\mathrm{mJ}$ in laser energy. For highpressure amplitudes, narrow pulse widths will be preferred because the far-field optoacoustic pressure is proportional to the time-derivative of the original laser pulse. The narrower temporal pulse also increases the operation frequency resulting in a tighter focus. In our configuration, the spatial-peak pulse-average (SPPA) acoustic intensity of the LGFU waveform shown in Fig. 2(a) was $\sim 4000 \mathrm{~W} /$ $\mathrm{cm}^{2}$ for laser energy of $12 \mathrm{~mJ} /$ pulse. However, the acoustic intensity decreases to $\sim 8 \mathrm{~mW} / \mathrm{cm}^{2}$ as we apply a temporal average (i.e. spatial-peak temporal-average; SPTA). This is due to the low repetition rate of the current laser pulses. For higher-intensity applications, lasers with higher-repetition rates are commercially available with the similar pulse energy (tens of $\mathrm{mJ})$ and temporal width $(5 \sim 8 \mathrm{~ns})$. For example, a pulse repetition of $2 \mathrm{kHz}$ would result in $>2 \mathrm{~W} / \mathrm{cm}^{2}$ in the SPTA with laser irradiation of $>30 \mathrm{~mJ} /$ pulse. This would accumulate significant heat at focal volumes. We note that the heating is an essential mechanism for thermal ablation-based therapy, but this is unfavorable in some other applications such as drug delivery and thrombolysis ${ }^{31,32}$. Especially in cell environments, a temperature change of only a few ${ }^{\circ} \mathrm{C}$ can cause transformation of the cellular metabolism.

We demonstrated the single-cell removal from the substrates and the surrounding cell networks, as an example of high-precision treatment which cannot be addressed by low-frequency, high-amplitude ultrasound. Leveraging the single-cell precision, we expect that our technique can be extended into delicate tissue structures and fine vasculatures as a means of a non-contact and non-thermal surgery. The LGFU-induced shock can directly break cell adhesion with the surrounding contacts. Moreover, as the micro-bubbles quickly grow and collapse at the targeted spot, these produce localized liquid jetstream and secondary shock waves. These become strong disruption sources to the cell in contact or from a distance of tens of $\mu \mathrm{m}$. We also note that the polymer film was used as a cell supporting layer. Therefore, it is also possible to destroy the polymer film underneath the cells which forms physical contacts. As the polymer is fallen off, the cells lose their sites to the substrates. Without the polymer supporting layer, the threshold pressure for the cell detachment will depend on specific adhesion strength of the cells to substrates as well as the substrate conditions to induce the cavitation in terms of acoustic impedance and surface topography.

In conclusion, we presented a new approach to optoacoustically generate high-frequency and high-amplitude focused ultrasound. Unprecedented magnitude of optoacoustic pressure was achieved due to efficient optoacoustic energy conversion enabled by nanocomposites of Au-coated CNTs and PDMS, the high-frequency nature of laser pulses, and the high focal gain from the low $f$-number lenses. The type I lens with 6-mm diameter was shown to generate a peak positive pressure amplitude of $>50 \mathrm{MPa}$ within a tight focal width of $75 \mu \mathrm{m}$ in lateral and $400 \mu \mathrm{m}$ in axial directions. The cavitation bubbles were tens of $\mu \mathrm{m}$ in dimensions and typical lifetime was shorter than $20 \mu \mathrm{s}$. We demonstrated applications of LGFU for noncontact targeted disruption in high precision: micro-scale fragmentation of solid materials and single-cell surgery. The dimensions of the disrupted area could be controlled from $6 \mu \mathrm{m}$ up to $400 \mu \mathrm{m}$ depending on the laser intensity and the incident LGFU amplitude. We 
demonstrated selective removal of a single cell from a cell-culture substrate and from neighboring cells with positional accuracy of $<25 \mu \mathrm{m}$. The LGFU has great flexibility in terms of transmitter designs and excitation laser choices to control ultrasonic frequencies, amplitudes, and intensities. As a result, we expect that the LGFU will become a versatile modality as a high-accuracy tool for ultrasonic therapy of cells, blood vessels, and tissue layers.

\section{Methods}

Material preparation. For growth of CNTs, we prepared fused silica substrates coated by catalyst layers of $\mathrm{Fe}(\sim 1 \mathrm{~nm})$ and $\mathrm{Al}_{2} \mathrm{O}_{3}(\sim 3 \mathrm{~nm})$ deposited by using a sputtering system. The fused silica substrates were plano-concave optical lenses (purchased from Edmund Optics, Barrington, NJ) with 5.5-mm radius-of-curvature and 6-mm diameter (type I lens), and $11.46 \mathrm{~mm}$ and $12 \mathrm{~mm}$ (type II lens), respectively. Multi-walled CNTs were grown in a mixture of $\mathrm{C}_{2} \mathrm{H}_{4} / \mathrm{H}_{2} / \mathrm{He}$ in an atmospheric pressure tube furnace at $775^{\circ} \mathrm{C}$. This process led to a tangled CNT layer that conformed to the curved surface of the lens. The as-grown CNTs, which have an optical extinction of $60 \sim 70 \%$, were then coated by a gold layer of $20 \mathrm{~nm}$. This further enhanced the optical extinction higher than $85 \%$ without increasing the overall source thickness significantly. Then, PDMS was spin-coated over the CNT-grown surface at 2000 r.p.m. for 2 minutes, and then cured at $100^{\circ} \mathrm{C}$ for 1 hour. We previously confirmed that the PDMS infiltrates the CNT network, forming a well-organized nano-composite film ${ }^{20}$.

Experimental configurations for temporal and spatial characterizations. Figure 1 shows the experimental schematic used for generation and characterization of the focused ultrasound. A 6-ns pulsed laser (Surelite I-20, Continuum, Santa Clara, CA) was used with a repetition rate of $20 \mathrm{~Hz}$. The laser beam initially has $5 \mathrm{~mm}$ in diameter. The laser beam was first attenuated by the neutral density filters and then expanded $(\times 5)$. The collimated beam was illuminated to the transparent (planar) side of the lens. The focused acoustic waves were detected by scanning the single-mode fiber-optic hydrophone $(6-\mu \mathrm{m}$ core and $125-\mu \mathrm{m}$ cladding in diameters) at the focal zone. Both the lens and the optical fiber were mounted on 3-dimensional motion stages for accurate alignment. The optical output was 3-dB coupled and transmitted to the photodetector. The photodetector has a broad electronic bandwidth over $75 \mathrm{MHz}$. The hydrophone operation is similar with that reported elsewhere ${ }^{33}$, but our fiber hydrophone has a significantly smaller active sensing diameter $(6-\mu \mathrm{m})$ which is suitable for measurement of the highly localized, high-frequency pressure field. Because of the finite aperture of the fiber, diffractive effects typically play a role in the frequency response, and a deconvolution of the waveform is required for such a probe. However, given that the lateral dimension of the LGFU focal spot is smaller than the fiber diameter, the diffractive effects are minimized. Then, the interaction of the incoming waves with the probe can be considered a pure reflection from an acoustically rigid surface for focal measurements. Based on this argument, the probe sensitivity was considered constant (i.e. doubled) over the bandwidth over $>15 \mathrm{MHz}$. By substitution comparison with a calibrated reference hydrophone, we obtained a sensitivity of $4.5 \mathrm{mV} / \mathrm{MPa}$ at $3.5 \mathrm{MHz}$ frequency. As this value is the result of $\sim 1.5$ fold enhancement due to the low-frequency diffraction effect ${ }^{26}$, we determined $6 \mathrm{mV} / \mathrm{MPa}$ as a final sensitivity of the current fiber-optic hydrophone. Both dc and ac signals were monitored by using a digital oscilloscope (WaveSurfer 432, LeCroy, Chestnut Ridge, NY). The waveforms in Fig. 3(a) are the result of averaging 20 signal traces in time-domain. For the passive detection measurement of the acoustic cavitation, we used a separate piezoelectric transducer with a center frequency of $15 \mathrm{MHz}$ (Model V319, Panametrics, Waltham, MA). The transducer output was directly recorded by using the digital oscilloscope.

High-speed camera monitoring and microscope setup. In order to capture the transient growth of cavitation, we used a high-speed camera (V210, Vision Research, Wayne, NJ, USA). It was integrated into an inverted optical microscope. The experimental schematic and description are provided in the supplementary material. For the polymer fragmentation experiment, the ultrasonic focus and the microscope view were fixed while we moved the polymer film on the microscope stage. For the cell experiment, the cultured cell substrates were moved to a petri-dish including the culture media on the microscope stage aligned with the LGFU. The bright-field and the fluorescence images of the cells were obtained in real time under the LGFU exposure.

Cell culture. SKOV3 human ovarian cancer cells were provided from Dr. Buckanovich's laboratory (University of Michigan, Ann Arbor, MI). They were initially seeded on glass slides spin-coated with PMMA (950K PMMA A4 (4\% solid contents) (Microchem, Newton, MA). Then, they were cultured in a Roswell Park Memorial Institute (RPMI) medium with $10 \%$ fetal bovine serum and $1 \%$ penicillin/streptomycin in a humidified incubator $\left(5 \% \mathrm{CO}_{2}, 37^{\circ} \mathrm{C}\right)$. Trypsin/ Ethylenediaminetetraacetic acid (EDTA) was used to re-suspend the cells in solution. These cells were diluted to $10^{6}$ cells $/ \mathrm{mL}$ and finally plated on the glass substrates spin-coated with the PMMA-based copolymer at 2000 r.p.m. for 30 seconds (from the solution of $4 \%$ by weight in tetrahydrofuran). Before the cell inoculation, the copolymer film was dried for 6 hours at $100^{\circ} \mathrm{C}$ to remove the solvent.

1. Hamilton, M. F. \& Blackstock, D. T. Nonlinear Acoustics, Acoustical Society of America, Melville (2008).

2. Coussios, C. C. \& Roy, R. A. Applications of Acoustics and Cavitation to Noninvasive Therapy and Drug Delivery. Annu. Rev. Fluid Mech. 40, 395-420 (2008).

3. Herbert, E., Balibar, S. \& Caupin, F. Cavitation pressure in water. Phys. Rev. E 74, 041603 (2006).

4. Filonenko, E. A. \& Khokhlova, V. A. Effect of acoustic nonlinearity on heating of biological tissue by high-intensity focused ultrasound. Acoust. Phys. 47, 468-475 (2001).

5. Mitragotri, S. Healing sound: the use of ultrasound in drug delivery and other therapeutic applications. Nat. Rev. Drug Discov. 4, 255-260 (2005).

6. Choi, J. J. et al. Noninvasive and localized blood-brain barrier disruption using focused ultrasound can be achieved at short pulse lengths and low pulse repetition frequencies. J. Cerebral Blood Flow Metabol. 31, 725-737 (2011).

7. Krasovitskia, B., Frenkelb, V., Shohama, S. \& Kimmela, E. Intramembrane cavitation as a unifying mechanism for ultrasound-induced bioeffects. Proc. Natl Acad. Sci. USA 108, 3258-3263 (2011).

8. Tufail, Y., Yoshihiro, A., Pati, S., Li, M. M. \& Tyler, W. J. Ultrasonic neuromodulation by brain stimulation with transcranial ultrasound. Nat. Protocols 6, 1453-1470 (2011).

9. Yoo, S.-S. et al. Focused ultrasound modulates region-specific brain activity. Neuroimage 56, 1267-1275 (2011).

10. Goldenstedt, C., Birer, A., Cathignol, D. \& Lafon, C. Blood clot disruption in vitro using shockwaves delivered by an extracorporeal generator after pre-exposure to lytic agent. Ultrasound Med. Biol. 35, 985-990 (2009).

11. Lingeman, J. E., McAteer, J. A., Gnessin, E. \& Eva, A. P. Shock wave lithotripsy: advances in technology and technique. Nat. Rev. Urology 6, 660-670 (2009).

12. Rassweiler, J. J. et al. Shock Wave Technology and Application: An Update. Eur. Urol. 59, 784-796 (2011).

13. Kennedy, J. E. High-intensity focused ultrasound in the treatment of solid tumours. Nat. Rev. Cancer 5, 321-327 (2005).

14. Zhou, Y.-F. High intensity focused ultrasound in clinical tumor ablation. World J. Clin. Oncol. 2, 8-27 (2011).

15. Dubinsky, T. J., Cuevas, C., Dighe, M. K., Kolokythas, O. \& Hwang, J. H. Highintensity focused ultrasound: current potential and oncologic applications. Am. J. Roentgenol. 190, 191-199 (2008).

16. Xu, Z. et al. Controlled ultrasound tissue erosion. IEEE Trans. Ultrason. Ferroelectr., Freq. Contr. 51, 726-736 (2004).

17. Apfel, R. E. \& Holland, C. K. Gauging the likelihood of cavitation from shortpulse, low-duty cycle diagnostic ultrasound. Ultrasound in Med. \& Biol. 17(2), 179-185 (1991).

18. Buma, T., Spisar, M. \& O’Donnell, M. High-frequency ultrasound array element using thermoelastic expansion in an elastomeric film. Appl. Phys. Lett. 79, 548-550 (2001)

19. Hou, Y., Kim, J. S., Ashkenazi, S., O’Donnell, M. \& Guo, L. J. Optical generation of high frequency ultrasound using two-dimensional gold nanostructure. Appl. Phys. Lett. 89, 093901 (2006)

20. Baac, H. W. et al. Carbon nanotube composite optoacoustic transmitters for strong and high frequency ultrasound generation. Appl. Phys. Lett. 97, 234104 (2010).

21. McKenzie, A. L. Physics of thermal processes in laser-tissue interaction. Phys Med. Biol. 35(9), 1175-1209 (1990).

22. Hart, A. J. \& Slocum, A. H. Rapid growth and flow-mediated nucleation of millimeter-scale aligned carbon nanotube structures from a thin-film catalyst. J. Phys. Chem. B 110, 8250-8257 (2006).

23. Cobbold, R. S. C. Foundations of Biomedical Ultrasound, Oxford University Press, New York (2007).

24. Diebold, G. J., Sun, T. \& Khan, M. I. Photoacoustic monopole radiation in one, two, and three dimensions. Phys. Rev. Lett. 67, 3384-3387 (1991).

25. Pelivanov, I. M., Kopylova, D. S., Podymova, N. B. \& Karabutov, A. A. Optoacoustic technique for thickness measurement of submicron metal coatings. Laser Phys. 19(6), 1350-1360 (2009).

26. Krücker, J. F. et al. Rigid piston approximation for computing the transfer function and angular response of a fiber-optic hydrophone. J. Acoust. Soc. Am. 107, 1994-2003 (2000)

27. Bailey, M. R. et al. Cavitation in shock wave lithotripsy. Fifth International Symposium on Cavitation, Cav03-OS-2-1-006, Osaka, Japan (2003).

28. Pishchalnikov, Y. A. et al. Cavitation bubble cluster activity in the breakage of kidney stones by lithotripter shockwaves. J. Endourol. 17, 435-446 (2003).

29. O'Donnell, M. et al. Optoacoustic generation of high frequency sound for 3-D ultrasonic imaging in medicine. Eur. Phys. J. Special Topics 153, 53-58 (2008).

30. Wu, N. et al. High-efficiency optical ultrasound generation using one-pot synthesized polydimethylsiloxane-gold nanoparticle nanocomposite. J. Opt. Soc. Am. B 29(8), 2016-2020 (2012)

31. Frenkel, V. \& Li, K. C. P. Potential role of pulsed-high intensity focused ultrasound in gene therapy. Future Oncol. 2, 111-119 (2006). 
32. Sakharov, D. V., Hekkenberg, R. T. \& Rijken, D. C. Acceleration of fibrinolysis by high-frequency ultrasound: the contribution of acoustic streaming and temperature rise. Thromb. Res. 100, 333-340 (2000).

33. Parsons, J. E., Cain, C. A. \& Fowlkes, J. B. Cost-effective assembly of a basic fiberoptic hydrophone for measurement of high-amplitude therapeutic ultrasound fields. J. Acoust. Soc. Am. 119, 1432-1440 (2006).

\section{Acknowledgements}

The authors would like to acknowledge the support from the National Science grant DMR 1120187, the National Institute of Health, and the University of Michigan. The authors also wish to acknowledge Simone Park for using the high-speed camera. H.W.B. acknowledges the support from the National Institute for International Education through Korean Ministry of Education, Science and Technology.

\section{Author contributions}

H.W.B. proposed the concept of the LGFU, designed and fabricated the devices, performed the experiments, and wrote the manuscript. J.G.O. contributed to the CNT growth and the lens fabrication. A.M. produced the fiber-optic hydrophone, contributed to the acoustic characterization of the LGFU, and edited the manuscript. K.-T.L. contributed to the characterization of the LGFU. Y.-C.C. cultured the cells and contributed to the single-cell detachment experiment. A.J.H. gave valuable comments on the CNT properties, and edited the manuscript. The CNT growth was performed in his laboratory. E.Y. gave valuable comments on the cell detachment experiment, and the cell culture was carried out in his laboratory. Z.X. gave valuable comments on the characterization of the LGFU and the acoustic cavitation, and edited the manuscript. Finally, L.J.G. supervised the overall work, contributed to the analysis of the experimental results, and edited the manuscript.

\section{Additional information}

Supplementary information accompanies this paper at http://www.nature.com/ scientificreports

Competing financial interests: The authors declare no competing financial interests.

License: This work is licensed under a Creative Commons

Attribution-NonCommercial-NoDerivs 3.0 Unported License. To view a copy of this license, visit http://creativecommons.org/licenses/by-nc-nd/3.0/

How to cite this article: Baac, H.W. et al. Carbon-Nanotube Optoacoustic Lens for Focused Ultrasound Generation and High-Precision Targeted Therapy. Sci. Rep. 2, 989; DOI:10.1038/srep00989 (2012). 